\title{
A Comparative Study of Space and Time Fractional KdV Equation through Analytical Approach with Nonlinear Auxiliary Equation
}

\author{
Hasibun Naher ${ }^{1, *}$, Humayra Shafia ${ }^{1}$, Md. Emran Ali $^{2}$, Gour Chandra Paul ${ }^{3}$ \\ ${ }^{1}$ Department of Mathematics and Natural Sciences, BRAC University, Bangladesh \\ ${ }^{2}$ Department of Textile Engineering, Northern University Bangladesh, Bangladesh \\ ${ }^{3}$ Department of Mathematics, University of Rajshahi, Bangladesh
}

Received September 23, 2019; Revised November 22, 2019; Accepted November 25, 2019

Copyright $\odot 2020$ by authors, all rights reserved. Authors agree that this article remains permanently open access under the terms of the Creative Commons Attribution License 4.0 International License

\begin{abstract}
In this article, the nonlinear partial fractional differential equation, namely the KdV equation is renewed with the help of modified Riemann- Liouville fractional derivative. The equation is transformed into the nonlinear ordinary differential equation by using the fractional complex transformation. The goal of this paper is to construct new analytical solutions of the space and time fractional nonlinear $\mathrm{KdV}$ equation through the extended $\left(G^{\prime} / G\right)$-expansion method. The work produces abundant exact solutions in terms of hyperbolic, trigonometric, rational, exponential, and complex forms, which are new and more general than existing results in literature. The newly generated solutions show that the executed method is a well-organized and competent mathematical tool to investigate a class of nonlinear evolution fractional order equations.
\end{abstract}

Keywords Complex Transformation, Modified Riemann-Liouville Derivative, New Extended $\left(G^{\prime} / G\right)$ -Expansion Method, Nonlinear Auxiliary Equation, Travelling Wave Solutions

\section{Introduction}

The application of fractional order differential equations is a matter of recent decades but the theory of fractional calculus has quite a long and prominent history. During the last three decades, fractional calculus has been applied to almost every field of science, namely mathematics, engineering and technology. Many important phenomena in turbulence, fluid dynamics, stochastic dynamical system, plasma physics, controlled thermonuclear fusion, non-linear control theory, image processing, non-linear biological systems, and astrophysics are well described by the fractional differential equations (FDEs) [1-7]. Therefore, one of the fundamental problems for these models is to engender traveling wave solutions for nonlinear FDEs (NLFDEs). In the literature various numerical and analytical methods have been developed such as the domain decomposition method [8], the differential transform method [9], the homotopy perturbation method [10], the first integral method [11], the fractional sub-equation method [12], the exp-function method [13,14], the $\left(G^{\prime} / G\right)$-expansion method [15-25] and so on.

As every nonlinear equation has its substantially noteworthy rich structure, still, significant research has to be done due to be well established the $\left(G^{\prime} / G\right)$-expansion method. It is important to present various new methods to construct many new exact solutions of FDEs. In this article, we would like to implement the new extended $\left(G^{\prime} / G\right)-$ expansion method with the help of modified Riemann Liouville derivative by Jumarie [26-28] to solve nonlinear space and time fractional $\mathrm{KdV}$ equation.

Let $f: R \rightarrow R, x \rightarrow f(x)$, be a continuous (but differentiable is not compulsory) function. The Jumarie's modified Riemann Liouville derivative of order $\alpha$ is as follows:

$$
D_{x}^{\alpha} f(x)=\left\{\begin{array}{lr}
\frac{1}{\Gamma(-\alpha)} \int_{0}^{x}(x-\xi)^{-\alpha-1}(f(\xi)-f(0)) d \xi, & \alpha<0 \\
\frac{1}{\Gamma(1-\alpha)} \frac{d}{d x} \int_{0}^{x}(x-\xi)^{-\alpha}(f(\xi)-f(0)) d \xi, & 0<\alpha<1 \\
\left(f^{(n)}(x)\right)^{(\alpha-n)} & n \leq \alpha<n+1, n \geq 1
\end{array}\right.
$$

We can have some important properties for the 
modified Riemann- Liouville derivatives are as [29]:

$$
\begin{gathered}
D_{x}^{\alpha} x^{r}=\frac{\Gamma(1+r)}{\Gamma(1+r-\alpha)} x^{r-\alpha}, r>0 \\
D_{x}^{\alpha}(f(x) g(x))=g(x) D_{x}^{\alpha} f(x)+f(x) D_{x}^{\alpha} g(x), \\
D_{x}^{\alpha} u[f(x)]=u_{f}^{\prime}(f) D_{x}^{\alpha} f(x)=D_{x}^{\alpha} u(f)\left(f_{x}^{\prime}\right)^{\alpha},
\end{gathered}
$$

where $\Gamma$ denotes the Gamma function.

\section{Illustration of the New Generalized $\left(G^{\prime} / G\right)$-Expansion Method for Fractional Partial Differential Equations}

The main steps of the suggested method are discussed in this section.

Let us consider the NLFDE as:

$P\left(\begin{array}{l}u, D_{t}^{\alpha} u, D_{x}^{\beta} u, D_{y}^{\gamma} u, D_{z}^{\delta} u, D_{t}^{\alpha} D_{t}^{\alpha} u, \\ D_{t}^{\alpha} D_{x}^{\beta} u, D_{x}^{\beta} D_{x}^{\beta} u, D_{x}^{\beta} D_{y}^{\gamma} u, D_{y}^{\gamma} D_{y}^{\gamma} u, \ldots\end{array}\right)=0,0<\alpha, \beta, \gamma, \delta<1$,

where $D_{t}^{\alpha}, D_{x}^{\alpha}, D_{x}^{\beta}, \ldots$ are the modified RiemannLiouville derivatives of $u, \quad u=u(x, y, t)$ is an unknown function, and $P$ is a polynomial of $u$ and its various partial fractional derivatives in which the highest order derivatives and nonlinear terms are involved.

The most important algorithm of the method is presented below:

Step 1. First, we convert the Fractional differential equation into an ordinary differential equation (ODE) using a fractional complex transformation [30,31]. Now, we define the traveling wave variable

$$
\begin{aligned}
& u(x, y, z, t)=u(\xi), \\
& \xi=\frac{K x^{\beta}}{\Gamma(\beta+1)}+\frac{N y^{\gamma}}{\Gamma(\gamma+1)}+\frac{M z^{\delta}}{\Gamma(\delta+1)}+\frac{L t^{\alpha}}{\Gamma(\alpha+1)},
\end{aligned}
$$

where $K, L, M$, and $N$ are nonzero arbitrary constants. Then we can rewrite Eq. (5) into an ODE of $u=u(\xi)$ in the form:

$$
Q\left(u, u^{\prime}, u^{\prime \prime}, u^{\prime \prime \prime}, \ldots\right)=0 .
$$

Where $Q$ is a polynomial of $u$ and primes denote the derivative with respect to $\xi$.

If possible, we can integrate Eq. (7) one or more times to get the outputs, where the constant of integration can be assumed to be zero for simplicity.
Step 2. Suppose that the travelling wave solution of Eq. (7) can be expressed as follows:

$$
u(\xi)=\sum_{j=0}^{m} a_{j}[\psi(\xi)]^{j}+\sum_{j=1}^{m} b_{j}[\psi(\xi)]^{-j},
$$

where $\psi(\xi)=[d+\varphi(\xi)]$ and $\varphi(\xi)$ is given by

$$
\varphi(\xi)=\left(G^{\prime}(\xi) / G(\xi)\right) .
$$

Here $a_{j}$ 's and $b_{j}$ 's and $d$ are unknown constants to be determined later and $G=G(\xi)$ satisfies the following auxiliary second order nonlinear ordinary differential equation (NLODE):

$$
A G G^{\prime \prime}-B G G^{\prime}-C\left(G^{\prime}\right)^{2}-E G^{2}=0,
$$

where $A, B, C$ and $E$ are real parameters.

Step 4. To determine the positive integer $m$ appearing in Eq. (8), we take the homogeneous balance between the highest order nonlinear term and the highest order derivative in Eq. (7).

Step 5. Substituting Eq. (8) and Eq. (10) along with Eq. (9) into Eq. (7) with the value of $m$ obtained in Step 4, we obtain polynomials in $(d+\varphi(\xi))^{m}(m=0,1,2, \ldots)$ and $(d+\varphi(\xi))^{-m}(m=1,2,3, \ldots)$. Then, setting each coefficient of the resulted polynomials to zero, a system of algebraic equations for $a_{j}(j=0,1,2, \ldots, m)$, $b_{j}(j=1,2, \ldots, m), K, L, M, N$ and $d \quad$ can be obtained.

Step 6. Suppose that the value of the constants can be found by solving the algebraic equations which are obtained in step 5 . Substituting the values of $a_{j}(j=0,1,2, \ldots, m)$,

$b_{j}(j=1,2, \ldots, m), K, L, M, N, d$ and the general solution of Eq. (10) into Eq. (8), we can obtain a variety of exact travelling wave solutions of Eq. (5).

Step 7. The general solutions of Eq. (10) are as follows [22]:

Family 1. When $B \neq 0, \lambda=A-C$ and $\Omega=B^{2}+4 E(A-C)>0$,

$$
\varphi(\xi)=\left(\frac{G^{\prime}}{G}\right)=\frac{B}{2 \lambda}+\frac{\sqrt{\Omega}}{2 \lambda} \frac{C_{1} \sinh \left(\frac{\sqrt{\Omega}}{2 \lambda} \xi\right)+C_{2} \cosh \left(\frac{\sqrt{\Omega}}{2 \lambda} \xi\right)}{C_{1} \cosh \left(\frac{\sqrt{\Omega}}{2 \lambda} \xi\right)+C_{2} \sinh \left(\frac{\sqrt{\Omega}}{2 \lambda} \xi\right)} .
$$

Family 2. When $B \neq 0, \lambda=A-C$ and $\Omega=B^{2}+4 E(A-C)<0$, 
$\varphi(\xi)=\left(\frac{G^{\prime}}{G}\right)=\frac{B}{2 \lambda}+\frac{\sqrt{-\Omega}}{2 \lambda} \frac{-C_{1} \sin \left(\frac{\sqrt{-\Omega}}{2 \lambda} \xi\right)+C_{2} \cos \left(\frac{\sqrt{-\Omega}}{2 \lambda} \xi\right)}{C_{1} \cos \left(\frac{\sqrt{-\Omega}}{2 \lambda} \xi\right)+C_{2} \sin \left(\frac{\sqrt{-\Omega}}{2 \lambda} \xi\right)}$.

Family 3. When $B \neq 0, \lambda=A-C$ and

$\Omega=B^{2}+4 E(A-C)=0$,

$$
\varphi(\xi)=\left(\frac{G^{\prime}}{G}\right)=\frac{B}{2 \lambda}+\frac{C_{2}}{C_{1}+C_{2} \xi} .
$$

Family 4. When $B=0, \lambda=A-C$ and $\Delta=\lambda E>0$,

$$
\varphi(\xi)=\left(\frac{G^{\prime}}{G}\right)=\frac{\sqrt{\Delta}}{\lambda} \frac{C_{1} \sinh \left(\frac{\sqrt{\Delta}}{\lambda} \xi\right)+C_{2} \cosh \left(\frac{\sqrt{\Delta}}{\lambda} \xi\right)}{C_{1} \cosh \left(\frac{\sqrt{\Delta}}{\lambda} \xi\right)+C_{2} \sinh \left(\frac{\sqrt{\Delta}}{\lambda} \xi\right)} .
$$

Family 5. $B=0, \lambda=A-C$ and $\Delta=\lambda E<0$,

$$
\varphi(\xi)=\left(\frac{G^{\prime}}{G}\right)=\frac{\sqrt{-\Delta}}{\lambda} \frac{-C_{1} \sin \left(\frac{\sqrt{-\Delta}}{\lambda} \xi\right)+C_{2} \cos \left(\frac{\sqrt{-\Delta}}{\lambda} \xi\right)}{C_{1} \cos \left(\frac{\sqrt{-\Delta}}{\lambda} \xi\right)+C_{2} \sin \left(\frac{\sqrt{-\Delta}}{\lambda} \xi\right)} .
$$

\section{Application of the Method}

The Korteweg-de Vries (KdV) equation is executed as a model to disclose a wide-range of physical phenomena for the enlargement and communication of nonlinear waves. Firstly, this equation was derived as an evolution equation with small amplitude, and long surface gravity waves propagation in shallow water channel [32]. Consequently, the $\mathrm{KdV}$ equation is present in various other physical contexts as collision-free hydromagnetic waves, stratified internal waves, ion-acoustic waves, particle acoustic waves, plasma physics, lattice dynamics and so on [33-36]. It is used as a model for solitons, turbulence, shock wave formation, boundary layer behavior and mass transport in fluid dynamics, and continuum mechanics [35,36]. Due to significant applications of non-integer order of the $\mathrm{KdV}$ equation it is required to be investigated for space and time fractional $\mathrm{KdV}$ equation. For example, the nonlinear oscillation of an earthquake could be modeled of the fractional derivatives [37].

Let the nonlinear $\mathrm{KdV}$ equation with space and time fractional derivatives [37]:

$$
\frac{\partial^{\alpha} u}{\partial t^{\alpha}}+a u \frac{\partial^{\beta} u}{\partial x^{\beta}}+\frac{\partial^{3 \beta} u}{\partial x^{3 \beta}}=0, t>0,0<\alpha, \beta \leq 1
$$

With the help of the fractional complex transformation

$$
u(x, t)=u(\xi) \text {, with } \xi=\frac{K x^{\beta}}{\Gamma(1+\beta)}+\frac{L t^{\alpha}}{\Gamma(1+\alpha)},
$$

where $K$ and $L$ constants, an ODE is obtained, which is on integrating twice, we obtain the following equation:

$$
\frac{1}{2} L u^{2}+\frac{a}{6} K u^{3}+\frac{K^{3}}{2}\left(u^{\prime}\right)^{2}+P_{1} u+P_{2}=0
$$

where $P_{1}$ and $P_{2}$ are integrating constants. Considering the homogeneous balance between the nonlinear term and the highest order derivative in Eq. (17), we have

$$
\begin{aligned}
& u(\xi)=a_{0}+a_{1} \psi(\xi)+a_{2}[\psi(\xi)]^{2} \\
& +b_{1}[\psi(\xi)]^{-1}+b_{2}[\psi(\xi)]^{-2},
\end{aligned}
$$

where $\psi(\xi)=[d+\varphi(\xi)], \quad \varphi(\xi)=G^{\prime}(\xi) / G(\xi)$.

And $a_{0}, a_{1}, a_{2}, b_{1}, b_{2}$ are constants to be evaluated later.

Using Eq. (18) along with Eq. (10) into Eq. (17), the left hand side results a polynomial in $(d+\varphi(\xi))^{m},(m=0,1,2, \ldots)$ and $(d+\varphi(\xi))^{-m},(m=1,2,3, \ldots)$. Taking each coefficient of these polynomials and setting them to zero, we have a set of algebraic equations (for simplicity the equations have not been displayed here) for $a_{0}, a_{1}, a_{2}, b_{1}, b_{2}, d, K, L, M, N$. Solving such algebraic equations using the symbolic computation software, such as Maple, we obtain following: 


\section{Case 1}

$$
\begin{aligned}
& L=\frac{-K}{A^{2}}\left\{12 K^{2} d^{2} \lambda^{2}+12 K^{2} B d \lambda-8 K^{2} E \lambda+a_{0} a A^{2}+K^{2} B^{2}\right\}, a_{1}=\frac{12 K^{2}}{a A^{2}}\left(2 d \lambda^{2}+B \lambda\right), \\
& d=d, a_{2}=\frac{-12 K^{2} \lambda^{2}}{a A^{2}}, b_{1}=0, b_{2}=0, p_{1}=\frac{K}{2 a A^{4}}\left(F_{1}+F_{2}+F_{3}+F_{4}+F_{5}+F_{6}\right), \\
& p_{2}=\frac{-K}{6 a^{2} A^{6}}\left(S_{1}+S_{2}+S_{3}+S_{4}+S_{5}+S_{6}+S_{7}+\ldots \ldots .+S_{16}\right),
\end{aligned}
$$

where $\lambda=A-C, F_{1}=144 K^{4} d^{4}\left(A^{4}+C^{4}\right)+a^{2} a_{0}^{2} A^{4}+a a_{0} K^{2} A^{2}\left(16 C E+24 C^{2} d^{2}-48 A C d^{2}\right)$,

$$
\begin{gathered}
F_{2}=576 K^{4} d^{2} A C E(A-C)-24 K^{4} B^{2} E(A-C)-96 K^{4} A C E^{2}+24 a a_{0} d K^{2} A^{3} B, \\
F_{3}=2 a a_{0} K^{2} A^{2} B^{2}-864 K^{4} d^{3} A B C(A-C)-192 d K^{4} B E\left(A^{2}-C^{2}\right)-336 d^{2} K^{4} A B^{2} C, \\
F_{4}=24 a a_{0} d^{2} K^{2} A^{4}-576 K^{4} d^{4} A C\left(A^{2}-C^{2}\right)-192 d^{2} K^{4} A^{3} E-288 d^{3} K^{4} B C^{3}+864 d^{4} K^{4} A^{2} C^{2}, \\
F_{5}=24 d K^{4} B^{3}(A-C)+168 K^{4} d^{2} B^{2}\left(A^{2}+C^{2}\right)+192 d^{2} K^{4} C^{3} E+288 d^{3} K^{4} B A^{3}, \\
F_{6}=384 d K^{4} A B C E-16 a a_{0} K^{2} A^{3} E+48 K^{4} E^{2}\left(A^{2}+C^{2}\right)-24 a a_{0} d K^{2} A^{2} B C,
\end{gathered}
$$

$S_{1}=432 K^{6} B^{2} C^{2} E^{2}+a^{3} a_{0}^{3} A^{6}+432 a a_{0} d^{4} K^{4} A^{2} C^{2}-288 a a_{0} K^{4} A^{3} C E^{2}-72 a a_{0} K^{4} A^{3} B^{2} E-576 a a_{0} d^{2} K^{4} A^{5} E$,

$S_{2}=2592 a a_{0} d^{4} K^{4} A^{4} C^{2}-1728 a a_{0} d^{4} K^{4} A^{3} C\left(A^{2}+C^{2}\right)+846 a a_{0} d^{3} K^{4} A^{5} B+504 a a_{0} d^{2} K^{4} A^{4} B^{2}+36 a^{2} a_{0}^{2} d^{2} K^{2} A^{4} C^{2}$,

$S_{3}=72 a a_{0} d K^{4} A^{3} B^{3}+24 a^{2} a_{0}^{2} K^{2} A^{4} C E-72 a^{2} a_{0}^{2} d^{2} K^{2} A^{5} C+36 a^{2} a_{0}^{2} d K^{2} A^{5} B+144 a a_{0} K^{4} A^{2} C^{2} E^{2}$,

$S_{4}=1152 a a_{0} d K^{4} A^{3} B C E-576 a a_{0} d K^{4} A^{2} B C^{2} E+432 a a_{0} d^{4} K^{4} A^{6}+36 a^{2} a_{0}^{2} d^{2} K^{2} A^{6}-24 a^{2} a_{0}^{2} K^{2} A^{5} E+3 a^{2} a_{0}^{2} K^{2} A^{4} B^{2}$,

$S_{5}=144 a a_{0} K^{4} A^{4} E^{2}+12960 d^{2} K^{6} A B^{2} C E(A-C)-41472 d^{3} K^{6} A^{2} B C^{2} E+5184 d K^{6} A B C^{2} E^{2}+27648 d^{3} K^{6} A B C^{3} E$,

$S_{6}=27648 d^{3} K^{6} A^{3} B C E-5184 d K^{6} A^{2} B C E^{2}+1728 d K^{6} A B^{3} C E+4320 d^{2} K^{6} B^{2} C^{3} E-6912 d K^{6} B C^{4} E-1728 d K^{6} B C^{3} E^{2}$,

$S_{7}=34560 d^{4} K^{6} A^{2} C^{3} E-17280 d^{4} K^{6} A C^{4} E-6912 d^{2} K^{6} A C^{3} E^{2}-22464 d^{4} K^{6} A B^{2} C^{3}-864 d K^{6} B^{3} C^{2} E-51840 d^{5} K^{6} A^{2} B C^{3}$,

$S_{8}=25920 d^{5} K^{6} A B C^{4}-34560 d^{4} K^{6} A^{3} C^{2} E+10368 d^{2} K^{6} A^{2} C^{2} E^{2}+33696 d^{4} K^{6} A^{2} B^{2} C^{2}+7776 d^{3} K^{6} A B^{3} C^{2}+51840 d^{5} K^{6} A^{3} B C^{2}$,

$S_{9}=17280 d^{4} K^{6} A^{4} C E-6912 d^{2} K^{6} A^{3} C E^{2}-864 d^{2} K^{6} A B^{4} C-22464 d^{4} K^{6} A^{3} B^{2} C-7776 d^{3} K^{6} A^{2} B^{3} C-864 K^{6} A B^{2} C E^{2}$,

$S_{10}=1728 d K^{6} A^{3} B E^{2}-25920 d^{5} K^{6} B C-4320 d^{2} K^{6} A^{3} B^{2}-6912 d^{3} K^{6} A^{4} B E-864 d K^{6} A^{2} B^{3} E+5616 d^{4} K^{6} B^{2} C^{4}$,

$S_{11}=3456 d^{4} K^{6} C^{5} E-25920 d^{3} K^{6} B^{3} C^{3}-5184 d^{5} K^{6} B C^{5}+1728 d^{2} K^{6} C^{4} E^{2}+25920 d^{6} K^{6} A^{4} C^{2}-10368 d^{6} K^{6} A C^{5}$, $S_{12}=432 d^{2} K^{6} B^{4} C^{2}+25920 d^{6} K^{6} A^{2} C^{4}-34560 d^{6} K^{6} A^{3} C^{3}-10368 d^{6} K^{6} A^{5} C-3456 d^{4} K^{6} A^{5} E+1728 d^{2} K^{6} A^{4} E^{2}$, $S_{13}=432 d^{2} K^{6} A^{2} B^{4}+5616 d^{4} K^{6} A^{4} B^{2}+2592 d^{3} K^{6} A^{3} B^{3}+432 K^{6} A^{2} B^{2} E^{2}+5184 d^{5} K^{6} A^{5} B+1728 d^{6} K^{6} C^{6}$, $S_{14}=1728 d^{6} K^{6} A^{6}+72 a a_{0} K^{4} A^{2} B^{2} C E+1728 a a_{0} d^{2} K^{4} A^{3} C E(A-C)+2592 a a_{0} d^{3} K^{4} A^{3} B C^{2}-2592 a a_{0} d^{3} K^{4} A^{4} B C$,

$S_{15}=576 a a_{0} d^{2} K^{4} A^{2} C^{3} E-576 a a_{0} d K^{4} A^{4} B E-1008 a a_{0} d^{2} K^{4} A^{3} B^{2} C-864 a a_{0} d^{3} K^{4} A^{2} B C^{3}$, $S_{16}=504 a a_{0} d^{2} K^{4} A^{2} B^{2} C^{2} 72 a a_{0} d K^{4} A^{2} B^{3} C-36 a^{2} a_{0}^{2} d K^{2} A^{4} B C$. 


\section{Case 2}

$$
\begin{aligned}
& L=\frac{-K}{A^{2}}\left\{12 K^{2} d^{2}(A-C)^{2}+12 K^{2} B d(A-C)-8 K^{2} E(A-C)+a_{0} a A^{2}+K^{2} B^{2}\right\}, a_{1}=0, \\
& a_{2}=0, d=d, b_{1}=\frac{12 K^{2}}{a A^{2}}\left\{2 d^{3}(A-C)^{2}+3 d^{2} B(A-C)-2 d E(A-C)+d B^{2}-B E\right\}, \\
& b_{2}=\frac{-12 K^{2}}{a A^{2}}\left\{d^{4}(A-C)^{2}+2 d^{3} B(A-C)-2 d^{2} E(A-C)+d^{2} B^{2}-2 d B E+E^{2}\right\}, \\
& p_{1}=\frac{K}{2 a A^{4}}\left(q_{1}+q_{2}+q_{3}+q_{4}+q_{5}+q_{6}\right), p_{2}=\frac{-K}{6 a^{2} A^{6}}\left(h_{1}+h_{2}+h_{3}+\ldots \ldots+h_{15}\right),
\end{aligned}
$$

where $q_{1}=144 K^{4} d^{4}\left(A^{4}+C^{4}\right)+a^{2} a_{0}^{2} A^{4}+a a_{0} K^{2} A^{2}\left(16 C E+24 C^{2} d^{2}-48 A C d^{2}\right)$,

$$
\begin{aligned}
& q_{2}=24 a a_{0} K^{2} d A^{3} B-96 K^{4} A C E^{2}-24 K^{4} B^{2} E(A-C)+576 d^{2} K^{4} A C E(A-C) \text {, } \\
& q_{3}=2 a a_{0} K^{2} A^{2} B^{2}-864 d^{3} K^{4} A B C(A-C)-192 d K^{4} B E\left(A^{2}+C^{2}\right)-336 d^{2} K^{4} A B^{2} C, \\
& q_{4}=24 a a_{0} d^{2} K^{2} A^{4}-192 d^{3} K^{4} A^{3} E-288 d^{3} K^{4} B C^{3}+864 d^{4} K^{4} A^{2} C^{2}-576 d^{4} K^{4} A C\left(A^{2}+C^{2}\right) \text {, } \\
& q_{5}=192 d^{2} K^{4} C^{3} E+288 d^{3} K^{4} B A^{3}+168 d^{2} K^{4} B^{2}\left(A^{2}+C^{2}\right)+24 d K^{4} B^{3}(A-C), \\
& q_{6}=384 d K^{4} A B C E-16 a a_{0} K^{2} A^{3} E+48 K^{4} E^{2}\left(A^{2}+C^{2}\right)-24 a a_{0} K^{2} A^{2} B C, \\
& h_{1}=432 K^{6} B^{2} C^{2} E^{2}+a^{3} a_{0}^{3} A^{6}+432 a a_{0} d^{2} K^{4} A^{2} C^{4}-a a_{0} K^{4} A^{3} E\left(288 E C+72 B^{2}\right)-576 a_{0} d^{2} K^{4} A^{5} E \text {, } \\
& h_{2}=a a_{0} d^{4} K^{4} A^{3} C\left\{2592 A C-1728 C\left(C^{2}+A^{2}\right)\right\}+a a_{0} d^{2} K^{4} A^{4} B(864 d A+504 A B)+36 a^{2} a_{0}^{2} d^{2} K^{2} A^{4} C^{2} \text {, } \\
& h_{3}=72 a a_{0} d K^{4} A^{3} B^{3}+a^{2} a_{0}^{2} K^{2} A^{4}\left(24 C E-72 d^{2} A C+36 d A B\right)+a a_{0} K^{4} A^{2} E C(144 E C+1152 d A B) \text {, } \\
& h_{4}=a a_{0} K^{4} A^{4}\left(144 E^{2}+432 d^{4} A^{2}\right)-576 a a_{0} d K^{4} A^{2} B C^{2} E+a^{2} a_{0}^{2} K^{2} A^{4}\left(36 d^{2} A^{2}-24 A E+3 B^{2}\right), \\
& h_{5}=d^{2} K^{6} A^{2} B C E(27648 d A+12960 B-41472 d C)+d K^{6} A B C^{2} E\left(27648 d^{2} C+5184 E-12960 d B\right) \text {, } \\
& h_{6}=d K^{6} A B C E\left(1728 B^{2}-5184 A E\right)-d^{3} K^{6} C^{4} E(6912 B+17280 d A)+d K^{6} B C^{3} E(4320 d B-1728 E) \text {, } \\
& h_{7}=d^{4} K^{6} A C^{3}\left(34560 A E-22464 B^{2}-51840 d A B+25920 d^{2} B C\right)-d K^{6} C^{2} E\left(6912 d A C E-864 B^{3}\right) \text {, } \\
& h_{8}=d^{4} K^{6} A^{2} C\left(33696 B^{2} C+51840 d A B C+17280 A^{2} E\right)+d^{2} K^{6} A C^{2}\left(10368 A E^{2}+7776 d d B^{3}-34560 d^{2} A^{2} E\right) \text {, } \\
& h_{9}=-d^{4} K^{6} A^{3} B C(22464 B+25920 d A)-d^{2} K^{6} A C\left(6912 A^{2} E^{2}+864 B^{4}+7776 d A B^{3}\right)-864 K^{6} A B^{2} C E^{2} \text {, } \\
& h_{10}=d^{3} K^{6} B^{2} C^{3}(5616 d C+2592 B)-d^{2} K^{6} A^{3} B E(4320 B+6912 d A)+d K^{6} A^{2} B E\left(1728 A E-864 B^{2}\right) \text {, } \\
& h_{11}=d^{6} K^{6} A C\left(25920 A^{3} C-10368 C^{4}\right)+d^{4} K^{6} C^{5}(3456 E-5184 d B)+d^{2} K^{6} C^{2}\left(1728 C^{2} E^{2}+432 B^{4}\right) \text {, } \\
& h_{12}=d^{2} K^{6} A^{2}\left(1728 A^{2} E^{2}+432 B^{4}\right)-d^{6} K^{6} A^{3} C\left(34560 C^{2}+10368 A^{2}\right)+d^{4} K^{6} A^{2}\left(25920 C^{4} E^{2}-3456 A^{3} E\right) \text {, } \\
& h_{13}=1728 d^{6} K^{6}\left(A^{6}+C^{6}\right)+d^{3} K^{6} A^{3} B\left(5616 d A B+2592 B^{2}+5184 d^{2} A^{2}\right)+432 K^{6} A^{2} B^{2} E^{2}, \\
& h_{14}=72 a a_{0} K^{4} A^{2} B^{2} C E+1728 a a_{0} d^{2} K^{4} A^{3} C E(A-C)-2592 a a_{0} d^{3} K^{4} A^{3} B C(A-C)-576 a a_{0} d K^{4} A^{4} B E \text {, } \\
& h_{15}=576 a a_{0} d^{2} K^{4} A^{2} C^{3} E-1008 a a_{0} d^{2} K^{4} A^{3} B^{2} C-864 a a_{0} d^{3} K^{4} A^{2} B C^{3}+504 a a_{0} d^{2} K^{4} A^{2} B^{2} C^{2}-72 a a_{0} d K^{4} A^{2} B^{3} C-36 a^{2} a_{0}^{2} d K A^{4} B C,
\end{aligned}
$$




\section{Case 3}

$$
\begin{aligned}
& L=\frac{-K}{A^{2}}\left\{a a_{0} A^{2}-2 K^{2} B^{2}-8 K^{2} E(A-C)\right\}, d=\frac{-B}{2(A-C)}, a_{1}=0, a_{2}=\frac{-12 K^{2}(A-C)^{2}}{a A^{2}}, \\
& b_{1}=0, b_{2}=\frac{-3 K^{2}\left\{16 E^{2}(A-C)+8 B^{2} E\right\}}{4 a A^{2}(A-C)}, p_{1}=\frac{K}{2 a A^{4}}\left(m_{1}+m_{2}\right), p_{2}=\frac{-K}{6 a^{2} A^{6}}\left(n_{1}+n_{2}+n_{3}\right),
\end{aligned}
$$

where $m_{1}=a^{2} a_{0}^{2} A^{4}-16 a a_{0} K^{2} A^{2} E(A-C)-96 K^{4} B^{2} E(A-C)$,

$$
\begin{gathered}
m_{2}=384 K^{2} A C E^{2}-192 K^{4} A^{2} E^{2}-4 a a_{0} K^{2} A^{2} B^{2}-12 K^{4} B^{4}-192 K^{4} C^{2} E^{2}, \\
n_{1}=a^{3} a_{0}^{3} A^{6}-24 a^{2} a_{0}^{2} K^{2} A^{4} E(A-C)-576 a a_{0} K^{4} A^{2} E^{2}(A-C)^{2}-6 a^{2} a_{0}^{2} K^{2} A^{4} B^{2}, \\
n_{2}=13824 K^{6} E^{3}(A-C)^{3}-288 a a_{0} K^{4} A^{2} B^{2} E(A-C)-36 a a_{0} K^{4} A^{2} B^{4}, \\
n_{3}=10368 K^{6} B^{2} E^{2}(A-C)^{2}-2592 K^{6} B^{4} E(A-C)+216 K^{6} B^{6} .
\end{gathered}
$$

\section{Case 4}

$$
\begin{aligned}
& L=\frac{-K}{A^{2}}\left\{a_{0} a A^{2}-8 K^{2} E(A-C)-2 K^{2} B^{2}\right\}, a_{1}=0, a_{2}=0, b_{1}=0, d=\frac{-B}{2(A-C)}, \\
& b_{2}=\frac{-3 K^{2}\left\{16 E^{2}(A-C)^{2}+8 B^{2} E(A-C)+B^{4}\right\}}{4 a A^{2}(A-C)^{2}}, \\
& p_{1}=\frac{K}{2 a A^{4}}\left(48 K^{4} E^{2}(A-C)^{2}+24 K^{4} B^{2} E(A-C)-16 a_{0} a K^{2} A^{2} E(A-C)+3 K^{4} B^{4}+a^{2} a_{0}^{2} A^{4}-4 a_{0} a K^{2} A^{2} B^{2}\right), \\
& p_{2}=\frac{-a_{0} K}{6 a A^{4}}\left(144 K^{4} E^{2}(A-C)^{2}+72 K^{4} B^{2} E(A-C)-24 a_{0} a K^{2} A^{2} E(A-C)+9 K^{4} B^{4}+a^{2} a_{0}^{2} A^{4}-6 a_{0} a K^{2} A^{2} B^{2}\right) .
\end{aligned}
$$

To derive the new solitary wave solutions through the hyperbolic functional form, substituting Eq. (19) into Eq. (18) along with Eq. (11), whenever $C_{1}=0$ but $C_{2} \neq 0$, and $C_{2}=0$ but $C_{1} \neq 0$, we have the following solutions:

$$
\begin{aligned}
& u 1_{1}(\xi)=a_{0}+\frac{12 K^{2}}{a A^{2}}\left(d^{2} \lambda^{2}+B d \lambda+\frac{B^{2}}{4}-\frac{\Omega}{4} \operatorname{coth}^{2}\left(\frac{\sqrt{\Omega}}{2 \lambda} \xi\right)\right), \\
& u 1_{2}(\xi)=a_{0}+\frac{12 K^{2}}{a A^{2}}\left(d^{2} \lambda^{2}+B d \lambda+\frac{B^{2}}{4}-\frac{\Omega}{4} \tanh ^{2}\left(\frac{\sqrt{\Omega}}{2 \lambda} \xi\right)\right),
\end{aligned}
$$

where $\xi=\frac{K x^{\beta}}{\Gamma(1+\beta)}-\frac{K t^{\alpha}}{A^{2} \Gamma(1+\alpha)}\left\{12 K^{2} d^{2} \lambda^{2}+12 K^{2} B d \lambda-8 K^{2} E \lambda+a_{0} a A^{2}+K^{2} B^{2}\right\}$.

After surrogating the complex transformation, we can turn out the following traveling wave solutions:

$$
\begin{gathered}
u 1_{11}(x, t)=a_{0}+\frac{12 K^{2}}{a A^{2}}\left(d^{2} \lambda^{2}+B d \lambda+\frac{B^{2}}{4}-\frac{\Omega}{4} \operatorname{coth}^{2}\left\{\frac{\sqrt{\Omega}}{2 \lambda}\left(\frac{K x^{\beta}}{\Gamma(1+\beta)}+\frac{L t^{\alpha}}{\Gamma(1+\alpha)}\right)\right\}\right), \\
u 1_{21}(\xi)=a_{0}+\frac{12 K^{2}}{a A^{2}}\left(d^{2} \lambda^{2}+B d \lambda+\frac{B^{2}}{4}-\frac{\Omega}{4} \tanh ^{2}\left\{\frac{\sqrt{\Omega}}{2 \lambda}\left(\frac{K x^{\beta}}{\Gamma(1+\beta)}+\frac{L t^{\alpha}}{\Gamma(1+\alpha)}\right)\right\}\right),
\end{gathered}
$$


where $L=\frac{-K}{A^{2}}\left\{12 K^{2} d^{2} \lambda^{2}+12 K^{2} B d \lambda-8 K^{2} E \lambda+a_{0} a A^{2}+K^{2} B^{2}\right\}$.

Furthermore, to achieve exact solutions in terms of trigonometric functional form, substituting Eq. (19) into Eq. (18) together with Eq. (12), and applying conditions (i) $C_{1}=0$ but $C_{2} \neq 0$, and (ii) $C_{2}=0$ but $C_{1} \neq 0$, we attain the following solutions:

$$
\begin{aligned}
& u 2_{1}(\xi)=a_{0}+\frac{12 K^{2}}{a A^{2}}\left(d^{2} \lambda^{2}+B d \lambda+\frac{B^{2}}{4}+\frac{\Omega}{4} \cot ^{2}\left(\frac{\sqrt{-\Omega}}{2 \lambda} \xi\right)\right), \\
& u 2_{2}(\xi)=a_{0}+\frac{12 K^{2}}{a A^{2}}\left(d^{2} \lambda^{2}+B d \lambda+\frac{B^{2}}{4}+\frac{\Omega}{4} \tan ^{2}\left(\frac{\sqrt{-\Omega}}{2 \lambda} \xi\right)\right),
\end{aligned}
$$

where $\xi=\frac{K x^{\beta}}{\Gamma(1+\beta)}+\frac{L t^{\alpha}}{\Gamma(1+\alpha)}, L=\frac{-K}{A^{2}}\left\{12 K^{2} d^{2} \lambda^{2}+12 K^{2} B d \lambda-8 K^{2} E \lambda+a_{0} a A^{2}+K^{2} B^{2}\right\}$.

Making use of the complex variable $\xi$, the solitary wave solutions turn into the following forms:

$$
\begin{aligned}
& u 2_{11}(x, t)=a_{0}+\frac{12 K^{2}}{a A^{2}}\left(d^{2} \lambda^{2}+B d \lambda+\frac{B^{2}}{4}+\frac{\Omega}{4} \cot ^{2}\left(\frac{i \sqrt{\Omega}}{2 \lambda}\left(\frac{K x^{\beta}}{\Gamma(1+\beta)}+\frac{L t^{\alpha}}{\Gamma(1+\alpha)}\right)\right)\right) \\
& u 2_{21}(x, t)=a_{0}+\frac{12 K^{2}}{a A^{2}}\left(d^{2} \lambda^{2}+B d \lambda+\frac{B^{2}}{4}+\frac{\Omega}{4} \tan ^{2}\left(\frac{i \sqrt{\Omega}}{2 \lambda}\left(\frac{K x^{\beta}}{\Gamma(1+\beta)}+\frac{L t^{\alpha}}{\Gamma(1+\alpha)}\right)\right)\right)
\end{aligned}
$$

where $L=\frac{-K}{A^{2}}\left\{12 K^{2} d^{2} \lambda^{2}+12 K^{2} B d \lambda-8 K^{2} E \lambda+a_{0} a A^{2}+K^{2} B^{2}\right\}$.

Taking into account Eq. (19) together with Eq. (13) into Eq. (18) and after simplifying the wave solutions become:

$$
u 3_{1}(\xi)=a_{0}+\frac{12 K^{2}}{a A^{2}}\left\{d^{2} \lambda^{2}+B d \lambda+\frac{B^{2}}{4}-\left(\frac{C_{2} \lambda}{C_{1}+C_{2} \xi}\right)^{2}\right\},
$$

where $\xi=\frac{K x^{\beta}}{\Gamma(1+\beta)}+\frac{L t^{\alpha}}{\Gamma(1+\alpha)}, \quad L=\frac{-K}{A^{2}}\left\{12 K^{2} d^{2} \lambda^{2}+12 K^{2} B d \lambda-8 K^{2} E \lambda+a_{0} a A^{2}+K^{2} B^{2}\right\}$.

$$
u 3_{11}(x, t)=a_{0}+\frac{12 K^{2}}{a A^{2}}\left\{d^{2} \lambda^{2}+B d \lambda+\frac{B^{2}}{4}-\left(\frac{C_{2} \lambda}{C_{1}+C_{2}\left(\frac{K x^{\beta}}{\Gamma(1+\beta)}+\frac{L t^{\alpha}}{\Gamma(1+\alpha)}\right)}\right)\right\}
$$

Setting up Eq.(19) together with Eq. (14) into Eq. (18) (if $C_{1}=0$ but $C_{2} \neq 0$, and $C_{2}=0$ but $C_{1} \neq 0$ ) and after simplifying, the wave solutions become:

$$
\begin{aligned}
& u 4_{1}(\xi)=a_{0}+\frac{12 K^{2}}{a A^{2}}\left(d^{2} \lambda^{2}-\Delta \operatorname{coth}^{2}\left(\frac{\sqrt{\Delta}}{\lambda} \xi\right)\right), \\
& u 4_{2}(\xi)=a_{0}+\frac{12 K^{2}}{a A^{2}}\left(d^{2} \lambda^{2}-\Delta \tanh ^{2}\left(\frac{\sqrt{\Delta}}{\lambda} \xi\right)\right),
\end{aligned}
$$

where $\xi=\frac{K x^{\beta}}{\Gamma(1+\beta)}+\frac{L t^{\alpha}}{\Gamma(1+\alpha)}$.

$$
u 4_{11}(x, t)=a_{0}+\frac{12 K^{2}}{a A^{2}}\left(d^{2} \lambda^{2}-\Delta \operatorname{coth}^{2}\left(\frac{\sqrt{\Delta}}{\lambda}\left(\frac{K x^{\beta}}{\Gamma(1+\beta)}+\frac{L t^{\alpha}}{\Gamma(1+\alpha)}\right)\right)\right),
$$




$$
u 4_{21}(x, t)=a_{0}+\frac{12 K^{2}}{a A^{2}}\left(d^{2} \lambda^{2}-\Delta \tanh ^{2}\left(\frac{\sqrt{\Delta}}{\lambda}\left(\frac{K x^{\beta}}{\Gamma(1+\beta)}+\frac{L t^{\alpha}}{\Gamma(1+\alpha)}\right)\right)\right),
$$

where $L=\frac{-K}{A^{2}}\left\{12 K^{2} d^{2} \lambda^{2}+12 K^{2} B d \lambda-8 K^{2} E \lambda+a_{0} a A^{2}+K^{2} B^{2}\right\}$.

According to the substitution of Eq. (19) together with Eq. (15) into Eq. (18) (if $C_{1}=0$ but $C_{2} \neq 0$, and $C_{2}=0$ but $C_{1} \neq 0$ ) and after simplifying, the wave solutions become:

$$
\begin{aligned}
& u 5_{1}(\xi)=a_{0}+\frac{12 K^{2}}{a A^{2}}\left(d^{2} \lambda^{2}+\Delta \cot ^{2}\left(\frac{i \sqrt{\Delta}}{\lambda} \xi\right)\right), \\
& u 5_{2}(\xi)=a_{0}+\frac{12 K^{2}}{a A^{2}}\left(d^{2} \lambda^{2}+\Delta \operatorname{tah}^{2}\left(\frac{i \sqrt{\Delta}}{\lambda} \xi\right)\right),
\end{aligned}
$$

where $\xi=\frac{K x^{\beta}}{\Gamma(1+\beta)}+\frac{L t^{\alpha}}{\Gamma(1+\alpha)}$.

$$
\begin{aligned}
& u 5_{11}(x, t)=a_{0}+\frac{12 K^{2}}{a A^{2}}\left(d^{2} \lambda^{2}+\Delta \cot ^{2}\left(\frac{i \sqrt{\Delta}}{\lambda}\left(\frac{K x^{\beta}}{\Gamma(1+\beta)}+\frac{L t^{\alpha}}{\Gamma(1+\alpha)}\right)\right)\right), \\
& u 5_{21}(x, t)=a_{0}+\frac{12 K^{2}}{a A^{2}}\left(d^{2} \lambda^{2}+\Delta \tan ^{2}\left(\frac{i \sqrt{\Delta}}{\lambda}\left(\frac{K x^{\beta}}{\Gamma(1+\beta)}+\frac{L t^{\alpha}}{\Gamma(1+\alpha)}\right)\right)\right),
\end{aligned}
$$

where $L=\frac{-K}{A^{2}}\left\{12 K^{2} d^{2} \lambda^{2}+12 K^{2} B d \lambda-8 K^{2} E \lambda+a_{0} a A^{2}+K^{2} B^{2}\right\}$.

Applying Eq. (20) into Eq. (18) together with Eq. (11) to (Eq. (15), respectively, we have the following simplified form various classical traveling wave solutions which have numerous significant applications:

$$
\begin{aligned}
& u 6_{1}(\xi)=a_{0}+b_{1}\left[d+\frac{B}{2 \lambda}+\frac{\sqrt{\Omega}}{2 \lambda} \frac{C_{1} \sinh \left(\frac{\sqrt{\Omega}}{2 \lambda} \xi\right)+C_{2} \cosh \left(\frac{\sqrt{\Omega}}{2 \lambda} \xi\right)}{C_{1} \cosh \left(\frac{\sqrt{\Omega}}{2 \lambda} \xi\right)+C_{2} \sinh \left(\frac{\sqrt{\Omega}}{2 \lambda} \xi\right)}\right]^{-1} \\
& +b_{2}\left[d+\frac{B}{2 \lambda}+\frac{\sqrt{\Omega}}{2 \lambda} \frac{C_{1} \sinh \left(\frac{\sqrt{\Omega}}{2 \lambda} \xi\right)+C_{2} \cosh \left(\frac{\sqrt{\Omega}}{2 \lambda} \xi\right)}{C_{1} \cosh \left(\frac{\sqrt{\Omega}}{2 \lambda} \xi\right)+C_{2} \sinh \left(\frac{\sqrt{\Omega}}{2 \lambda} \xi\right)}\right]^{-2}, \\
& u 7_{1}(\xi)=a_{0}+b_{1}\left[d+\left(G^{\prime} / G\right)\right]^{-1}+b_{2}\left[d+\left(G^{\prime} / G\right)\right]^{-2} \text {, } \\
& \text { where }\left(\frac{G^{\prime}}{G}\right)=\frac{B}{2 \lambda}+\frac{\sqrt{-\Omega}}{2 \lambda} \frac{-C_{1} \sin \left(\frac{\sqrt{-\Omega}}{2 \lambda} \xi\right)+C_{2} \cos \left(\frac{\sqrt{-\Omega}}{2 \lambda} \xi\right) \text {, }}{C_{1} \cos \left(\frac{\sqrt{-\Omega}}{2 \lambda} \xi\right)+C_{2} \sin \left(\frac{\sqrt{-\Omega}}{2 \lambda} \xi\right)} \\
& u 8_{1}(\xi)=a_{0}+b_{1}\left[d+\frac{B}{2 \lambda}+\frac{C_{2}}{C_{1}+C_{2} \xi}\right]^{-1}+b_{2}\left[d+\frac{B}{2 \lambda}+\frac{C_{2}}{C_{1}+C_{2} \xi}\right]^{-2},
\end{aligned}
$$




$$
\begin{aligned}
& u 9_{1}(\xi)=a_{0}+b_{1}\left[d+\frac{\sqrt{\Delta}}{\lambda} \frac{C_{1} \sinh \left(\frac{\sqrt{\Delta}}{\lambda} \xi\right)+C_{2} \cosh \left(\frac{\sqrt{\Delta}}{\lambda} \xi\right)}{C_{1} \cosh \left(\frac{\sqrt{\Delta}}{\lambda} \xi\right)+C_{2} \sinh \left(\frac{\sqrt{\Delta}}{\lambda} \xi\right)}\right]^{-1} \\
& +b_{2}\left[d+\frac{\sqrt{\Delta}}{\lambda} \frac{C_{1} \sinh \left(\frac{\sqrt{\Delta}}{\lambda} \xi\right)+C_{2} \cosh \left(\frac{\sqrt{\Delta}}{\lambda} \xi\right)}{C_{1} \cosh \left(\frac{\sqrt{\Delta}}{\lambda} \xi\right)+C_{2} \sinh \left(\frac{\sqrt{\Delta}}{\lambda} \xi\right)}\right]^{-2}, \\
& u 10_{1}(\xi)=a_{0}+b_{1}\left[d+\frac{\sqrt{-(A-C) E}}{\lambda} \frac{C_{1} \sin \left(\frac{i \sqrt{(A-C) E}}{\lambda} \xi\right)+C_{2} \cos \left(\frac{i \sqrt{(A-C) E}}{\lambda} \xi\right)}{C_{1} \cos \left(\frac{i \sqrt{(A-C) E}}{\lambda} \xi\right)+C_{2} \sin \left(\frac{i \sqrt{(A-C) E}}{\lambda} \xi\right)}\right]^{-1} \\
& +b_{2}\left[d+\frac{\sqrt{-(A-C) E}}{\lambda} \frac{C_{1} \sin \left(\frac{i \sqrt{(A-C) E}}{\lambda} \xi\right)+C_{2} \cos \left(\frac{i \sqrt{(A-C) E}}{\lambda} \xi\right)}{C_{1} \cos \left(\frac{i \sqrt{(A-C) E}}{\lambda} \xi\right)+C_{2} \sin \left(\frac{i \sqrt{(A-C) E}}{\lambda} \xi\right)}\right]^{-2},
\end{aligned}
$$

where

$$
\begin{gathered}
\xi=\frac{K x^{\beta}}{\Gamma(1+\beta)}+\frac{L t^{\alpha}}{\Gamma(1+\alpha)}, L=\frac{-K}{A^{2}}\left\{12 K^{2} d^{2}(A-C)^{2}+12 K^{2} B d(A-C)-8 K^{2} E(A-C)+a_{0} a A^{2}+K^{2} B^{2}\right\} \\
b_{1}=\frac{12 K^{2}}{a A^{2}}\left\{2 d^{3}(A-C)^{2}+3 d^{2} B(A-C)-2 d E(A-C)+d B^{2}-B E\right\}, \\
b_{2}=\frac{-12 K^{2}}{a A^{2}}\left\{d^{4}(A-C)^{2}+2 d^{3} B(A-C)-2 d^{2} E(A-C)+d^{2} B^{2}-2 d B E+E^{2}\right\} .
\end{gathered}
$$

Now making use of the complex variable $\xi$, the above classical solitary wave solutions turn into the following form: If $C_{2}=0, C_{1} \neq 0$ :

$$
\begin{aligned}
u 6_{11}(x, t)= & a_{0}+b_{1}\left[d+\frac{B}{2 \lambda}+\frac{\sqrt{\Omega}}{2 \lambda} \tanh \frac{\sqrt{\Omega}}{2 \lambda}\left(\frac{K x^{\beta}}{\Gamma(1+\beta)}+\frac{L t^{\alpha}}{\Gamma(1+\alpha)}\right)\right]^{-1} \\
& +b_{2}\left[d+\frac{B}{2 \lambda}+\frac{\sqrt{\Omega}}{2 \lambda} \tanh \frac{\sqrt{\Omega}}{2 \lambda}\left(\frac{K x^{\beta}}{\Gamma(1+\beta)}+\frac{L t^{\alpha}}{\Gamma(1+\alpha)}\right)\right]^{-2},
\end{aligned}
$$

Now considering $C_{1}=0, C_{2} \neq 0$, we have the following form:

$$
\begin{aligned}
& u 6_{12}(x, t)=a_{0}+b_{1}\left[d+\frac{B}{2 \lambda}+\frac{\sqrt{\Omega}}{2 \lambda}\left\{\frac{\left.e^{\frac{\sqrt{\Omega}}{2 \lambda}\left(\frac{K x^{\beta}}{\Gamma(1+\beta)}+\frac{L t^{\alpha}}{\Gamma(1+\alpha)}\right)}+e^{-\frac{\sqrt{\Omega}}{2 \lambda}\left(\frac{K x^{\beta}}{\Gamma(1+\beta)}+\frac{L t^{\alpha}}{\Gamma(1+\alpha)}\right)}\right)}{\left.e^{\frac{\sqrt{\Omega}}{2 \lambda}\left(\frac{K x^{\beta}}{\Gamma(1+\beta)}+\frac{L t^{\alpha}}{\Gamma(1+\alpha)}\right)}-e^{-\frac{\sqrt{\Omega}}{2 \lambda}\left(\frac{K x^{\beta}}{\Gamma(1+\beta)}+\frac{L t^{\alpha}}{\Gamma(1+\alpha)}\right)}\right)}\right]^{-1}\right. \\
& +b_{2}\left[d+\frac{B}{2 \lambda}+\frac{\sqrt{\Omega}}{2 \lambda}\left\{\frac{\left.e^{\frac{\sqrt{\Omega}}{2 \lambda}\left(\frac{K x^{\beta}}{\Gamma(1+\beta)}+\frac{L t^{\alpha}}{\Gamma(1+\alpha)}\right)}+e^{-\frac{\sqrt{\Omega}}{2 \lambda}\left(\frac{K x^{\beta}}{\Gamma(1+\beta)}+\frac{L t^{\alpha}}{\Gamma(1+\alpha)}\right)}\right)}{\left.e^{\frac{\sqrt{\Omega}}{2 \lambda}\left(\frac{K x^{\beta}}{\Gamma(1+\beta)}+\frac{L t^{\alpha}}{\Gamma(1+\alpha)}\right)}-e^{-\frac{\sqrt{\Omega}}{2 \lambda}\left(\frac{K x^{\beta}}{\Gamma(1+\beta)}+\frac{L t^{\alpha}}{\Gamma(1+\alpha)}\right)}\right)}\right]^{-2},\right.
\end{aligned}
$$

If $C_{2}=0, C_{1} \neq 0$ : 


$$
\begin{aligned}
& u 7_{11}(x, t)=a_{0}+b_{1}\left[d+\frac{B}{2 \lambda}-\frac{i \sqrt{\Omega}}{2 \lambda}\left\{\frac{e^{\frac{i \sqrt{-\Omega}}{2 \lambda}\left(\frac{K x^{\beta}}{\Gamma(1+\beta)}+\frac{L t^{\alpha}}{\Gamma(1+\alpha)}\right)}-e^{-\frac{i \sqrt{-\Omega}}{2 \lambda}\left(\frac{K x^{\beta}}{\Gamma(1+\beta)}+\frac{L t^{\alpha}}{\Gamma(1+\alpha)}\right)}}{e^{\frac{i \sqrt{-\Omega}}{2 \lambda}\left(\frac{K x^{\beta}}{\Gamma(1+\beta)}+\frac{L t^{\alpha}}{\Gamma(1+\alpha)}\right)}+e^{-\frac{i \sqrt{-\Omega}}{2 \lambda}\left(\frac{K x^{\beta}}{\Gamma(1+\beta)}+\frac{L t^{\alpha}}{\Gamma(1+\alpha)}\right)}}\right\}\right]^{-1} \\
& +b_{2}\left[d+\frac{B}{2 \lambda}-\frac{i \sqrt{\Omega}}{2 \lambda}\left\{\frac{e^{\frac{i \sqrt{-\Omega}}{2 \lambda}\left(\frac{K x^{\beta}}{\Gamma(1+\beta)}+\frac{L t^{\alpha}}{\Gamma(1+\alpha)}\right)}-e^{-\frac{i \sqrt{-\Omega}}{2 \lambda}\left(\frac{K x^{\beta}}{\Gamma(1+\beta)}+\frac{L t^{\alpha}}{\Gamma(1+\alpha)}\right)}}{e^{\frac{i \sqrt{-\Omega}}{2 \lambda}\left(\frac{K x^{\beta}}{\Gamma(1+\beta)}+\frac{L t^{\alpha}}{\Gamma(1+\alpha)}\right)}+e^{-\frac{i \sqrt{-\Omega}}{2 \lambda}\left(\frac{K x^{\beta}}{\Gamma(1+\beta)}+\frac{L t^{\alpha}}{\Gamma(1+\alpha)}\right)}}\right\}\right]^{-2},
\end{aligned}
$$

Now considering $C_{1}=0, C_{2} \neq 0$, we have

$$
\begin{aligned}
& u 7_{12}(x, t)=a_{0}+ b_{1}\left[d+\frac{B}{2 \lambda}+\frac{i \sqrt{\Omega}}{2 \lambda} \cot \left\{\frac{i \sqrt{\Omega}}{2 \lambda}\left(\frac{K x^{\beta}}{\Gamma(1+\beta)}+\frac{L t^{\alpha}}{\Gamma(1+\alpha)}\right)\right\}\right]^{-1} \\
&+b_{2}\left[d+\frac{B}{2 \lambda}-\frac{i \sqrt{\Omega}}{2 \lambda} \cot \left\{\frac{i \sqrt{\Omega}}{2 \lambda}\left(\frac{K x^{\beta}}{\Gamma(1+\beta)}+\frac{L t^{\alpha}}{\Gamma(1+\alpha)}\right)\right]\right]^{-2}, \\
& u 8_{11}(x, t)=a_{0}+b_{1}\left[d+\frac{B}{2 \lambda}+\frac{C_{2}}{C_{1}+C_{2}\left(\frac{K x^{\beta}}{\Gamma(1+\beta)}+\frac{L t^{\alpha}}{\Gamma(1+\alpha)}\right)}\right]^{-1} \\
&+ b_{2}\left[d+\frac{B}{2 \lambda}+\frac{C_{1}+C_{2}\left(\frac{K x^{\beta}}{\Gamma(1+\beta)}+\frac{L t^{\alpha}}{\Gamma(1+\alpha)}\right)}{C^{\prime}}\right]^{-2},
\end{aligned}
$$

If $C_{2}=0, C_{1} \neq 0$, we have

$$
\begin{aligned}
u 9_{11}(x, t)=a_{0} & +b_{1}\left[d+\sqrt{\frac{E}{A-C}} \tanh \left\{\sqrt{\frac{E}{A-C}}\left(\frac{K x^{\beta}}{\Gamma(1+\beta)}+\frac{L t^{\alpha}}{\Gamma(1+\alpha)}\right)\right]\right]^{-1} \\
& +b_{2}\left[d+\sqrt{\frac{E}{A-C}} \tanh \left\{\sqrt{\frac{E}{A-C}}\left(\frac{K x^{\beta}}{\Gamma(1+\beta)}+\frac{L t^{\alpha}}{\Gamma(1+\alpha)}\right)\right]\right]^{-2},
\end{aligned}
$$

$C_{1}=0, C_{2} \neq 0$, 


$$
\begin{aligned}
& u 9_{12}(x, t)=a_{0}+b_{1}\left[d+\sqrt{\frac{E}{A-C}}\left\{\frac{\left.e^{\frac{\sqrt{\Delta}}{\lambda}\left(\frac{K x^{\beta}}{\Gamma(1+\beta)}+\frac{L t^{\alpha}}{\Gamma(1+\alpha)}\right)}+e^{\frac{-\sqrt{\Delta}}{\lambda}\left(\frac{K x^{\beta}}{\Gamma(1+\beta)}+\frac{L t^{\alpha}}{\Gamma(1+\alpha)}\right)}\right)}{\left.e^{\frac{\sqrt{\Delta}}{\lambda}\left(\frac{K x^{\beta}}{\Gamma(1+\beta)}+\frac{L t^{\alpha}}{\Gamma(1+\alpha)}\right)}-e^{\frac{-\sqrt{\Delta}}{\lambda}\left(\frac{K x^{\beta}}{\Gamma(1+\beta)}+\frac{L t^{\alpha}}{\Gamma(1+\alpha)}\right)}\right)}\right\}\right]^{-1} \\
& +b_{2}\left[d+\sqrt{\frac{E}{A-C}}\left\{\frac{e^{\frac{\sqrt{\Delta}}{\lambda}\left(\frac{K x^{\beta}}{\Gamma(1+\beta)}+\frac{L t^{\alpha}}{\Gamma(1+\alpha)}\right)}+e^{\frac{-\sqrt{\Delta}}{\lambda}\left(\frac{K x^{\beta}}{\Gamma(1+\beta)}+\frac{L t^{\alpha}}{\Gamma(1+\alpha)}\right)}}{\left.e^{\frac{\sqrt{\Delta}}{\lambda}\left(\frac{K x^{\beta}}{\Gamma(1+\beta)}+\frac{L t^{\alpha}}{\Gamma(1+\alpha)}\right)}-e^{\frac{-\sqrt{\Delta}}{\lambda}\left(\frac{K x^{\beta}}{\Gamma(1+\beta)}+\frac{L t^{\alpha}}{\Gamma(1+\alpha)}\right)}\right\}}\right]^{-2},\right.
\end{aligned}
$$

If $C_{2}=0, C_{1} \neq 0$, we have

$$
\begin{aligned}
u 10_{11}(x, t)= & a_{0}+b_{1}\left[d-\frac{i \sqrt{\Delta}}{\lambda} \tan \left\{\frac{i \sqrt{\Delta}}{\lambda}\left(\frac{K x^{\beta}}{\Gamma(1+\beta)}+\frac{L t^{\alpha}}{\Gamma(1+\alpha)}\right)\right\}\right]^{-1} \\
& +b_{2}\left[d-\frac{i \sqrt{\Delta}}{\lambda} \tan \left\{\frac{i \sqrt{\Delta}}{\lambda}\left(\frac{K x^{\beta}}{\Gamma(1+\beta)}+\frac{L t^{\alpha}}{\Gamma(1+\alpha)}\right)\right]\right]^{-2},
\end{aligned}
$$

$C_{1}=0, C_{2} \neq 0$

$$
u 10_{12}(x, t)=a_{0}+b_{1}\left[d+\frac{\sum_{n=0}^{\infty}(2 n+1)}{\left(\frac{K x^{\beta}}{\Gamma(1+\beta)}+\frac{L t^{\alpha}}{\Gamma(1+\alpha)}\right)}\right]^{-1}+b_{2}\left[d+\frac{\sum_{n=0}^{\infty}(2 n+1)}{\left(\frac{K x^{\beta}}{\Gamma(1+\beta)}+\frac{L t^{\alpha}}{\Gamma(1+\alpha)}\right)}\right]^{-2},
$$

where

$$
\begin{aligned}
& L=\frac{-K}{A^{2}}\left\{12 K^{2} d^{2}(A-C)^{2}+12 K^{2} B d(A-C)-8 K^{2} E(A-C)+a_{0} a A^{2}+K^{2} B^{2}\right\} \\
& b_{1}=\frac{12 K^{2}}{a A^{2}}\left\{2 d^{3}(A-C)^{2}+3 d^{2} B(A-C)-2 d E(A-C)+d B^{2}-B E\right\}, \\
& b_{2}=\frac{-12 K^{2}}{a A^{2}}\left\{d^{4}(A-C)^{2}+2 d^{3} B(A-C)-2 d^{2} E(A-C)+d^{2} B^{2}-2 d B E+E^{2}\right\} .
\end{aligned}
$$

Applying Eq. (21) into Eq. (18) together with Eq. (11) to (Eq. (15), respectively, we have the following simplified form various classical traveling wave solutions which have numerous significant applications:

$$
\begin{gathered}
u 11_{1}(\xi)=a_{0}-\frac{3 \Omega K^{2}}{a A^{2}}\left[\left\{\frac{C_{1} \sinh \left(\frac{\sqrt{\Omega}}{2 \lambda} \xi\right)+C_{2} \cosh \left(\frac{\sqrt{\Omega}}{2 \lambda} \xi\right)}{C_{1} \cosh \left(\frac{\sqrt{\Omega}}{2 \lambda} \xi\right)+C_{2} \sinh \left(\frac{\sqrt{\Omega}}{2 \lambda} \xi\right)}\right\}^{2}+\frac{8 \lambda\left(2 \lambda E^{2}+E B^{2}\right)}{\Omega^{2}}\left\{\frac{C_{1} \sinh \left(\frac{\sqrt{\Omega}}{2 \lambda} \xi\right)+C_{2} \cosh \left(\frac{\sqrt{\Omega}}{2 \lambda} \xi\right)}{C_{1} \cosh \left(\frac{\sqrt{\Omega}}{2 \lambda} \xi\right)+C_{2} \sinh \left(\frac{\sqrt{\Omega}}{2 \lambda} \xi\right)}\right\},\right. \\
u 12_{1}(\xi)=a_{0}+\frac{3 \Omega K^{2}}{a A^{2}}\left[\left\{\frac{C_{1} \sin \left(\frac{i \sqrt{\Omega}}{2 \lambda} \xi\right)+C_{2} \cos \left(\frac{i \sqrt{\Omega}}{2 \lambda} \xi\right)}{C_{1} \cos \left(\frac{i \sqrt{\Omega}}{2 \lambda} \xi\right)+C_{2} \sin \left(\frac{i \sqrt{\Omega}}{2 \lambda} \xi\right)}\right]^{2}\right\}+\frac{8 \lambda\left(2 \lambda E^{2}+E B^{2}\right)}{\Omega^{2}}\left\{\frac{C_{1} \sin \left(\frac{i \sqrt{\Omega}}{2 \lambda} \xi\right)+C_{2} \cos \left(\frac{i \sqrt{\Omega}}{2 \lambda} \xi\right)}{C_{1} \cos \left(\frac{i \sqrt{\Omega}}{2 \lambda} \xi\right)+C_{2} \sin \left(\frac{i \sqrt{\Omega}}{2 \lambda} \xi\right)}\right\}, \\
u 13_{1}(\xi)=a_{0}-\frac{12 K^{2}}{a A^{2}}\left[\left(\frac{\lambda C_{2}}{C_{1}+C_{2} \xi}\right)^{2}+\left(\frac{2 \lambda E^{2}+E B^{2}}{2 \lambda}\right)\left(\frac{C_{1}+C_{2} \xi}{C_{2}}\right)^{2}\right],
\end{gathered}
$$




$$
\begin{gathered}
u 14_{1}(\xi)=a_{0}-\frac{12 K^{2}}{a A^{2}}\left[\Delta\left\{\frac{C_{1} \sinh \left(\frac{\sqrt{\Delta}}{\lambda} \xi\right)+C_{2} \cosh \left(\frac{\sqrt{\Delta}}{\lambda} \xi\right)}{C_{1} \cosh \left(\frac{\sqrt{\Delta}}{\lambda} \xi\right)+C_{2} \sinh \left(\frac{\sqrt{\Delta}}{\lambda} \xi\right)}\right\}+\frac{\left(2 \lambda^{2} E^{2}+\lambda E B^{2}\right)}{2 \Delta}\left\{\frac{C_{1} \sinh \left(\frac{\sqrt{\Delta}}{\lambda} \xi\right)+C_{2} \cosh \left(\frac{\sqrt{\Delta}}{\lambda} \xi\right)}{C_{1} \cosh \left(\frac{\sqrt{\Delta}}{\lambda} \xi\right)+C_{2} \sinh \left(\frac{\sqrt{\Delta}}{\lambda} \xi\right)}\right]^{-2}\right], \\
u 15_{1}(\xi)=a_{0}+\frac{12 K^{2} \Delta}{a A^{2}}\left[\left\{\frac{-C_{1} \sin \left(\frac{\sqrt{-\Delta}}{\lambda} \xi\right)+C_{2} \cos \left(\frac{\sqrt{-\Delta}}{\lambda} \xi\right)}{C_{1} \cos \left(\frac{\sqrt{-\Delta}}{\lambda} \xi\right)+C_{2} \sin \left(\frac{\sqrt{-\Delta}}{2 \lambda} \xi\right)}\right\}^{2}+\frac{\lambda\left(2 \lambda E^{2}+E B^{2}\right)}{\Delta^{2}}\left\{\frac{-C_{1} \sin \left(\frac{\sqrt{-\Delta}}{\lambda} \xi\right)+C_{2} \cos \left(\frac{\sqrt{-\Delta}}{\lambda} \xi\right)}{C_{1} \cos \left(\frac{\sqrt{-\Delta}}{\lambda} \xi\right)+C_{2} \sin \left(\frac{\sqrt{-\Delta}}{2 \lambda} \xi\right)}\right\},\right.
\end{gathered}
$$

\section{Complex Forms:}

If $C_{2}=0, C_{1} \neq 0$, we have

$$
u 11_{11}(x, t)=a_{0}-\frac{3 \Omega K^{2}}{a A^{2}}\left[\left(p-\frac{p^{3}}{3}+\frac{2 p^{5}}{15}-\frac{17 p^{7}}{315}+\ldots\right)^{2}+\left\{\frac{8 \lambda\left(2 \lambda E^{2}+E B^{2}\right)}{\Omega^{2}}\right\}\left(p-\frac{p^{3}}{3}+\frac{2 p^{5}}{15}-\frac{17 p^{7}}{315}+\ldots\right)^{-2}\right],
$$

where $p=\frac{\sqrt{\Omega}}{2 \lambda}\left(\frac{K x^{\beta}}{\Gamma(1+\beta)}+\frac{L t^{\alpha}}{\Gamma(1+\alpha)}\right)$, and $L=\frac{-K}{A^{2}}\left\{a a_{0} A^{2}-2 K^{2} B^{2}-8 K^{2} E(A-C)\right\}$.

Now considering $C_{1}=0, C_{2} \neq 0$, we have the solutions having the following form:

$$
u 11_{12}(x, t)=a_{0}-\frac{3 \Omega K^{2}}{a A^{2}}\left[\left(\frac{e^{p}+e^{-p}}{e^{p}-e^{-p}}\right)^{2}+\left(\frac{e^{p}-e^{-p}}{e^{p}+e^{-p}}\right)^{2}\right],
$$

where $p=\frac{\sqrt{\Omega}}{2 \lambda}\left(\frac{K x^{\beta}}{\Gamma(1+\beta)}+\frac{L t^{\alpha}}{\Gamma(1+\alpha)}\right)$, and $L=\frac{-K}{A^{2}}\left\{a a_{0} A^{2}-2 K^{2} B^{2}-8 K^{2} E(A-C)\right\}$.

If $C_{2}=0, C_{1} \neq 0$ :

$$
\begin{aligned}
& u 7_{11}(x, t)=a_{0}+b_{1}\left[d+\frac{B}{2 \lambda}-\frac{i \sqrt{\Omega}}{2 \lambda}\left\{\frac{e^{\frac{i \sqrt{-\Omega}}{2 \lambda}\left(\frac{K x^{\beta}}{\Gamma(1+\beta)}+\frac{L t^{\alpha}}{\Gamma(1+\alpha)}\right)}-e^{-\frac{i \sqrt{-\Omega}}{2 \lambda}\left(\frac{K x^{\beta}}{\Gamma(1+\beta)}+\frac{L t^{\alpha}}{\Gamma(1+\alpha)}\right)}}{\left.e^{\frac{i \sqrt{-\Omega}}{2 \lambda}\left(\frac{K x^{\beta}}{\Gamma(1+\beta)}+\frac{L t^{\alpha}}{\Gamma(1+\alpha)}\right)}+e^{-\frac{i \sqrt{-\Omega}}{2 \lambda}\left(\frac{K x^{\beta}}{\Gamma(1+\beta)}+\frac{L t^{\alpha}}{\Gamma(1+\alpha)}\right)}\right)}\right\}\right]^{-1} \\
& +b_{2}\left[d+\frac{B}{2 \lambda}-\frac{i \sqrt{\Omega}}{2 \lambda}\left\{\frac{e^{\frac{i \sqrt{-\Omega}}{2 \lambda}\left(\frac{K x^{\beta}}{\Gamma(1+\beta)}+\frac{L t^{\alpha}}{\Gamma(1+\alpha)}\right)}-e^{-\frac{i \sqrt{-\Omega}}{2 \lambda}\left(\frac{K x^{\beta}}{\Gamma(1+\beta)}+\frac{L t^{\alpha}}{\Gamma(1+\alpha)}\right)}}{e^{\frac{i \sqrt{-\Omega}}{2 \lambda}\left(\frac{K x^{\beta}}{\Gamma(1+\beta)}+\frac{L t^{\alpha}}{\Gamma(1+\alpha)}\right)}+e^{-\frac{i \sqrt{-\Omega}}{2 \lambda}\left(\frac{K x^{\beta}}{\Gamma(1+\beta)}+\frac{L t^{\alpha}}{\Gamma(1+\alpha)}\right)}}\right\}\right]^{-2},
\end{aligned}
$$

Now considering $C_{1}=0, C_{2} \neq 0$, we have the following solutions:

$$
\begin{aligned}
u 7_{12}(x, t)=a_{0}+ & b_{1}\left[d+\frac{B}{2 \lambda}+\frac{i \sqrt{\Omega}}{2 \lambda} \cot \left\{\frac{i \sqrt{\Omega}}{2 \lambda}\left(\frac{K x^{\beta}}{\Gamma(1+\beta)}+\frac{L t^{\alpha}}{\Gamma(1+\alpha)}\right)\right\}\right]^{-1} \\
& +b_{2}\left[d+\frac{B}{2 \lambda}-\frac{i \sqrt{\Omega}}{2 \lambda} \cot \left\{\frac{i \sqrt{\Omega}}{2 \lambda}\left(\frac{K x^{\beta}}{\Gamma(1+\beta)}+\frac{L t^{\alpha}}{\Gamma(1+\alpha)}\right)\right]\right]^{-2},
\end{aligned}
$$




$$
\begin{aligned}
u 8_{11}(x, t)=a_{0}+b_{1}\left[d+\frac{B}{2 \lambda}+\frac{C_{2}}{C_{1}+C_{2}\left(\frac{K x^{\beta}}{\Gamma(1+\beta)}+\frac{L t^{\alpha}}{\Gamma(1+\alpha)}\right)}\right]^{-1} \\
+b_{2}\left[d+\frac{B}{2 \lambda}+\frac{C_{2}}{C_{1}+C_{2}\left(\frac{K x^{\beta}}{\Gamma(1+\beta)}+\frac{L t^{\alpha}}{\Gamma(1+\alpha)}\right)}\right]^{-2},
\end{aligned}
$$

If $C_{2}=0, C_{1} \neq 0$ :

$$
\begin{aligned}
u 9_{11}(x, t)=a_{0} & +b_{1}\left[d+\sqrt{\frac{E}{A-C}} \tanh \left\{\sqrt{\frac{E}{A-C}}\left(\frac{K x^{\beta}}{\Gamma(1+\beta)}+\frac{L t^{\alpha}}{\Gamma(1+\alpha)}\right)\right]\right]^{-1} \\
& +b_{2}\left[d+\sqrt{\frac{E}{A-C}} \tanh \left\{\sqrt{\frac{E}{A-C}}\left(\frac{K x^{\beta}}{\Gamma(1+\beta)}+\frac{L t^{\alpha}}{\Gamma(1+\alpha)}\right)\right]\right]^{-2},
\end{aligned}
$$

$C_{1}=0, C_{2} \neq 0$

$$
\begin{aligned}
& u 9_{12}(x, t)=a_{0}+b_{1}\left[d+\sqrt{\frac{E}{A-C}}\left\{\frac{e^{\frac{\sqrt{\Delta}}{\lambda}\left(\frac{K x^{\beta}}{\Gamma(1+\beta)}+\frac{L t^{\alpha}}{\Gamma(1+\alpha)}\right)}+e^{\frac{-\sqrt{\Delta}}{\lambda}\left(\frac{K x^{\beta}}{\Gamma(1+\beta)}+\frac{L t^{\alpha}}{\Gamma(1+\alpha)}\right)}}{e^{\frac{\sqrt{\Delta}}{\lambda}\left(\frac{K x^{\beta}}{\Gamma(1+\beta)}+\frac{L t^{\alpha}}{\Gamma(1+\alpha)}\right)}-e^{\frac{-\sqrt{\Delta}}{\lambda}\left(\frac{K x^{\beta}}{\Gamma(1+\beta)}+\frac{L t^{\alpha}}{\Gamma(1+\alpha)}\right)}}\right\}\right]^{-1} \\
& +b_{2}\left[d+\sqrt{\frac{E}{A-C}}\left\{\frac{e^{\frac{\sqrt{\Delta}}{\lambda}\left(\frac{K x^{\beta}}{\Gamma(1+\beta)}+\frac{L t^{\alpha}}{\Gamma(1+\alpha)}\right)}+e^{\frac{-\sqrt{\Delta}}{\lambda}\left(\frac{K x^{\beta}}{\Gamma(1+\beta)}+\frac{L t^{\alpha}}{\Gamma(1+\alpha)}\right)}}{e^{\frac{\sqrt{\Delta}}{\lambda}\left(\frac{K x^{\beta}}{\Gamma(1+\beta)}+\frac{L t^{\alpha}}{\Gamma(1+\alpha)}\right)}-e^{\frac{-\sqrt{\Delta}}{\lambda}\left(\frac{K x^{\beta}}{\Gamma(1+\beta)}+\frac{L t^{\alpha}}{\Gamma(1+\alpha)}\right)}}\right\}\right]^{-2},
\end{aligned}
$$

If $C_{2}=0, C_{1} \neq 0$, we have

$$
\begin{aligned}
u 10_{11}(x, t)= & a_{0}+b_{1}\left[d-\frac{i \sqrt{\Delta}}{\lambda} \tan \left\{\frac{i \sqrt{\Delta}}{\lambda}\left(\frac{K x^{\beta}}{\Gamma(1+\beta)}+\frac{L t^{\alpha}}{\Gamma(1+\alpha)}\right)\right]\right]^{-1} \\
& \left.+b_{2}\left[d-\frac{i \sqrt{\Delta}}{\lambda} \tan \left\{\frac{i \sqrt{\Delta}}{\lambda}\left(\frac{K x^{\beta}}{\Gamma(1+\beta)}+\frac{L t^{\alpha}}{\Gamma(1+\alpha)}\right)\right]\right\}\right]^{-2},
\end{aligned}
$$

If $C_{1}=0, C_{2} \neq 0$, we have

$$
u 10_{12}(x, t)=a_{0}+b_{1}\left[d+\frac{\sum_{n=0}^{\infty}(2 n+1)}{\left(\frac{K x^{\beta}}{\Gamma(1+\beta)}+\frac{L t^{\alpha}}{\Gamma(1+\alpha)}\right)}\right]^{-1}+b_{2}\left[d+\frac{\sum_{n=0}^{\infty}(2 n+1)}{\left(\frac{K x^{\beta}}{\Gamma(1+\beta)}+\frac{L t^{\alpha}}{\Gamma(1+\alpha)}\right)}\right]^{-2},
$$


where

$$
\begin{aligned}
& L=\frac{-K}{A^{2}}\left\{12 K^{2} d^{2}(A-C)^{2}+12 K^{2} B d(A-C)-8 K^{2} E(A-C)+a_{0} a A^{2}+K^{2} B^{2}\right\} \\
& b_{1}=\frac{12 K^{2}}{a A^{2}}\left\{2 d^{3}(A-C)^{2}+3 d^{2} B(A-C)-2 d E(A-C)+d B^{2}-B E\right\}, \\
& b_{2}=\frac{-12 K^{2}}{a A^{2}}\left\{d^{4}(A-C)^{2}+2 d^{3} B(A-C)-2 d^{2} E(A-C)+d^{2} B^{2}-2 d B E+E^{2}\right\} .
\end{aligned}
$$

It is important to note that the obtained solutions $u 1_{11}(x, t)$ to $u 10_{12}(x, t)$ of the fractional KdV equation with many arbitrary parameters are resourceful and most of those were not found in the earlier literature. The differential equation involving derivatives of non-integer orders can be suitable models for several complex physical phenomena, for this purpose the renowned FKdV equation was investigated. And these solutions might be implemented for solid state physics, electromagnetic interactions, electrodynamics, and quantum relativistic etc.

\section{Results and Discussion}

It is very crucial to mentioning that some of the newly constructed solutions disclose excellent similarity subject to particular values of arbitrary parameters with solutions those are already available in the earlier literature. A comparison

\begin{tabular}{|c|c|}
\hline Gepreel and Omran's solutions [37] & Our obtained solutions in this research \\
\hline $\begin{array}{l}\text { 1.When } \lambda^{2}-4 \mu>0, B=0 \text {, the solution is } \\
u(\xi)=a_{0}+\frac{3 K^{2} \lambda^{2}}{a}-\frac{3 K^{2}\left(\lambda^{2}-4 \mu\right)}{a} \operatorname{coth}^{2}\left\{\frac{\left(\lambda^{2}-4 \mu\right)}{2} \xi\right\}\end{array}$ & $\begin{array}{l}\text { 1.When } A=1, B=-\lambda, C=0, E=-\mu, B \neq 0, \quad \lambda=A-C, \\
\Omega=B^{2}+4 E(A-C)>0 \text {, the solution } \\
u 1_{1}(\xi)=a_{0}+\frac{12 K^{2}}{a A^{2}}\left(d^{2} \lambda^{2}+B d \lambda+\frac{B^{2}}{4}-\frac{\Omega}{4} \operatorname{coth}^{2}\left(\frac{\sqrt{\Omega}}{2 \lambda} \xi\right)\right)\end{array}$ \\
\hline $\begin{array}{l}\text { 2.When } \lambda^{2}-4 \mu<0, B=0 \text { the solution is } \\
u(\xi)=a_{0}+\frac{3 K^{2} \lambda^{2}}{a}-\frac{3 K^{2}\left(4 \mu-\lambda^{2}\right)}{a} \tan ^{2}\left\{\frac{i\left(\lambda^{2}-4 \mu\right)}{2} \xi\right\}\end{array}$ & $\begin{array}{l}\text { 2.When } A=1, B=-\lambda, C=0, E=-\mu, B \neq 0, \lambda=A-C, \\
\Omega=B^{2}+4 E(A-C)<0 \text {, the solution } \\
u 2_{2}(\xi)=a_{0}+\frac{12 K^{2}}{a A^{2}}\left(d^{2} \lambda^{2}+B d \lambda+\frac{B^{2}}{4}+\frac{\Omega}{4} \tan ^{2}\left(\frac{i \sqrt{\Omega}}{2 \lambda} \xi\right)\right)\end{array}$ \\
\hline $\begin{array}{l}\text { 3. When } \lambda^{2}-4 \mu>0, A=0 \text {, the solution is } \\
u(\xi)=a_{0}+\frac{3 K^{2} \lambda^{2}}{a}-\frac{3 K^{2}\left(\lambda^{2}-4 \mu\right)}{a} \tanh ^{2}\left\{\frac{\left(\lambda^{2}-4 \mu\right)}{2} \xi\right.\end{array}$ & $\begin{array}{l}\text { 3.When } A=1, B=-\lambda, C=0, E=-\mu, B \neq 0, \lambda=A-C, \\
\Omega=B^{2}+4 E(A-C)>0 \text {, the solution } \\
u 1_{2}(\xi)=a_{0}+\frac{12 K^{2}}{a A^{2}}\left(d^{2} \lambda^{2}+B d \lambda+\frac{B^{2}}{4}-\frac{\Omega}{4} \tanh ^{2}\left(\frac{\sqrt{\Omega}}{2 \lambda} \xi\right.\right.\end{array}$ \\
\hline
\end{tabular}
between Gepreel and Omran’s [37] solutions and our obtained solutions has been presented in the following table.

Table 1: Comparison between Gepreel and Omran's [37] solutions and obtained solutions in this research work

Beside these solutions, we obtained many new exact wave solutions from $u 4_{11}(x, t)$ to $u 10_{12}(x, t)$ in this work, and those were not being reported in the literature earlier. The produced solutions can become special function if the arbitrary constants take specific values. 


\section{Conclusions}

In this research, the nonlinear fractional complex transformation has been implemented to convert a certain fractional partial differential equations into another ODEs of integer order. The results are presented by a polynomial in $\left(G^{\prime} / G\right)$, where $\left(G^{\prime} / G\right)$ satisfies the second order nonlinear ODE as $A G G^{\prime \prime}-B G G^{\prime}-C\left(G^{\prime}\right)^{2}-E G^{2}=0$. We have examined the space and time fractional nonlinear $\mathrm{KdV}$ equation. As a result, we have produced new and further general solitary wave solutions such as single soliton, double solitons, kink type solutions, periodic solutions in terms of various hyperbolic forms, several trigonometric forms, rational forms etc., having many arbitrary parameters, which may have remarkable applications in applied mathematics and biological sciences. The new generalized $\left(G^{\prime} / G\right)$ method has its own advantage: direct, simple, succinct and can be executed for many other fractional nonlinear equations.

\section{REFERENCES}

[1] Dzieliński, A., Sierociuk, D., \& Sarwas, G. (2010). Some applications of fractional order calculus. Bulletin of the Polish Academy of Sciences: Technical Sciences, 58(4), 583-592.

[2] Luchko, Y. (2015). Wave-diffusion dualism of the neutral-fractional processes. Journal of Computational Physics, 293, 40-52.

[3] Lakshmikantham, V., \& Vatsala, A. S. (2008). Basic theory of fractional differential equations. Nonlinear Analysis: Theory, Methods \& Applications, 69(8), 2677-2682.

[4] Miller, K. S., \& Ross, B. (1993). An introduction to the fractional calculus and fractional differential equations.

[5] Diethelm, K., \& Ford, N. J. (2002). Analysis of fractional differential equations. Journal of Mathematical Analysis and Applications, 265(2), 229-248.

[6] Podlubny, I. (1998). Fractional differential equations: an introduction to fractional derivatives, fractional differential equations, to methods of their solution and some of their applications (Vol. 198). Academic press.

[7] Hilfer, R. (Ed.). (2000). Applications of fractional calculus in physics (Vol. 128). Singapore: World Scientific.

[8] Daftardar-Gejji, V., \& Jafari, H. (2005). Adomian decomposition: a tool for solving a system of fractional differential equations. Journal of Mathematical Analysis and Applications, 301(2), 508-518.

[9] Arikoglu, A., \& Ozkol, I. (2007). Solution of fractional differential equations by using differential transform method. Chaos, Solitons \& Fractals, 34(5), 1473-1481.

[10] Momani, S., \& Odibat, Z. (2007). Homotopy perturbation method for nonlinear partial differential equations of fractional order. Physics Letters A, 365(5), 345-350.

[11] Lu, B. (2012). The first integral method for some time fractional differential equations. Journal of Mathematical Analysis and Applications, 395(2), 684-693.

[12] Tang, B., He, Y., Wei, L., \& Zhang, X. (2012). A generalized fractional sub-equation method for fractional differential equations with variable coefficients. Physics Letters A, 376(38), 2588-2590.

[13] He, J. H. (2013). Exp-function method for fractional differential equations. International Journal of Nonlinear Sciences and Numerical Simulation, 14(6), 363-366.

[14] H. Naher, F. A. Abdullah, M. A. Akbar, New traveling wave solutions of the higher dimensional nonlinear partial differential equation by the Exp-function method, J. Appl. Math., Article ID 575387, 14 pp. doi: 10.1155(2012)575387.

[15] M. Wang, X. Li, J. Zhang, The $\left(G^{\prime} / G\right)$-expansion method and travelling wave solutions of nonlinear evolution equations in mathematical physics, Phys. Lett. A, 372 (2008): 417-423.

[16] E. M. E. Zayed. Traveling wave solutions for higher dimensional nonlinear evolution equations using the $\left(G^{\prime} / G\right)$-expansion method. J. Appl. Math. \& Informatics, 28(1-2) (2010): 383-395.

[17] H. Naher, F.A. Abdullah and M.A. Akbar. The $\left(G^{\prime} / G\right)$ -expansion method for abundant traveling wave solutions of Caudrey-Dodd-Gibbon equation. Math. Probl. Engr. 2011, 11. http://dx.doi.org/10.1155/(2011)/218216.

[18] H. Naher and F. A. Abdullah, Some new traveling wave solutions of the nonlinear reaction diffusion equation by using the improved $\left(G^{\prime} / G\right)$-expansion method, Math. Prob. Eng., Article ID 871724, 17 pp. doi:10.1155(2012) 871724.

[19] H. Naher and F. A. Abdullah. The improved $\left(G^{\prime} / G\right)$-expansion method to the $(2+1)$-dimensional breaking soliton equation. Journal of Computational Analysis \& Applications, 16(2) ( 2014): 220-235.

[20] M. A. Akbar, N. H. M. Ali and E. M. E. Zayed. A generalized and improved $\left(G^{\prime} / G\right)$-expansion method for nonlinear evolution equations, Math. Prob. Eng., Article ID 459879, 22 pp. doi:10.1155(2012)459879.

[21] H. Naher, F. A. Abdullah and M. A. Akbar, Generalized and Improved $\left(\mathrm{G}^{\prime} / \mathrm{G}\right)$-Expansion Method for 1)-Dimensional Modified KdV-Zakharov-Kuznetsev Equation, PloS one, 8(5) (2013): e64618.

[22] H. Naher, and F. A. Abdullah, New Approach of $\left(G^{\prime} / G\right)$ expansion method and new approach of generalized $\left(G^{\prime} / G\right)$-expansion method for nonlinear evolution equation AIP Advances, 3, 032116 (2013); doi: 10.1063/1.4794947.

[23] Bin, Z. (2012). (G'/G)-expansion method for solving fractional partial differential equations in the theory of 
mathematical physics. Communications in Theoretical Physics, 58(5), 623.

[24] Bekir, A., \& Güner, Ö. (2013). Exact solutions of nonlinear fractional differential equations by $\left(\mathrm{G}^{\prime} / \mathrm{G}\right)$-expansion method. Chinese Physics B, 22(11), 110202.

[25] Gepreel, K. A., \& Omran, S. (2012). Exact solutions for nonlinear partial fractional differential equations. Chinese Physics B, 21(11), 110204.

[26] Jumarie, G. (2006). Modified Riemann-Liouville derivative and fractional Taylor series of nondifferentiable functions further results. Computers \& Mathematics with Applications, 51(9-10), 1367-1376.

[27] Jumarie, G. (2007). Fractional partial differential equations and modified Riemann-Liouville derivative new methods for solution. Journal of Applied Mathematics and Computing, 24(1-2), 31-48.

[28] Jumarie, G. (2009). Table of some basic fractional calculus formulae derived from a modified Riemann-Liouville derivative for non-differentiable functions. Applied Mathematics Letters, 22(3), 378-385.

[29] Roy, R., Akbar, M. A., \& Wazwaz, A. M. (2018). Exact wave solutions for the nonlinear time fractional SharmaTasso-Olver equation and the fractional Klein-Gordon equation in mathematical physics. Optical and Quantum Electronics, 50(1), 25.

[30] Li, Z. B., \& He, J. H. (2011). Application of the fractional complex transform to fractional differential equations. Nonlinear Science Letters A-Mathematics, Physics and Mechanics, 2(3), 121-126.

[31] Li, Z. B., \& He, J. H. (2010). Fractional complex transform for fractional differential equations. Mathematical and Computational Applications, 15(5), 970-973.

[32] Korteweg, D. J., \& De Vries, G. (1895). XLI. On the change of form of long waves advancing in a rectangular canal, and on a new type of long stationary waves. The London, Edinburgh, and Dublin Philosophical Magazine and Journal of Science, 39(240), 422-443.

[33] Schamel, H. (1973). A modified Korteweg-de Vries equation for ion acoustic wavess due to resonant electrons. Journal of Plasma Physics, 9(3), 377-387.

[34] Wazwaz, A. M. (2008). New sets of solitary wave solutions to the $\mathrm{KdV}, \mathrm{mKdV}$, and the generalized $\mathrm{KdV}$ equations. Communications in Nonlinear Science and Numerical Simulation, 13(2), 331-339.

[35] El-Wakil, S. A., Abulwafa, E. M., Zahran, M. A., \& Mahmoud, A. A. (2011). Time-fractional KdV equation: formulation and solution using variational methods. Nonlinear Dynamics, 65(1-2), 55-63.

[36] Zafar, A. (2019). The expa function method and the conformable time-fractional $\mathrm{KdV}$ equations. Nonlinear Engineering, 8(1), 728-732.

[37] Gepreel, K. A., \& Omran, S. (2012). Exact solutions for nonlinear partial fractional differential equations. Chinese Physics B, 21(11), 110204. 